\title{
Large-scale cosmic ray anisotropy measured by the
} GRAPES-3 experiment

\section{Chakraborty, ${ }^{a, *}$ S. Ahmad, ${ }^{c}$ A. Chandra, ${ }^{c}$ S.R. Dugad, ${ }^{a}$ U.D. Goswami, ${ }^{l}$}

S.K. Gupta, ${ }^{a}$ B. Hariharan, ${ }^{a}$ Y. Hayashi, ${ }^{b}$ P. Jagadeesan, ${ }^{a}$ A. Jain, ${ }^{a}$ P. Jain, ${ }^{d}$ S. Kawakami, ${ }^{b}$ H. Kojima, ${ }^{e}$ S. Mahapatra, ${ }^{i}$ P.K. Mohanty, ${ }^{a}$ R. Moharana,${ }^{j}$ Y. Muraki, ${ }^{g}$ P.K. Nayak, ${ }^{a}$ T. Nonaka, ${ }^{h}$ A. Oshima, ${ }^{e}$ B.P. Pant, ${ }^{j}$ D. Pattanaik, ${ }^{a, i}$ G.S. Pradhan, ${ }^{k}$ P.S. Rakshe, ${ }^{a}$ M. Rameez, ${ }^{a}$ K. Ramesh, ${ }^{a}$ L.V. Reddy, ${ }^{a}$ R. Sahoo, ${ }^{k}$ R. Scaria, ${ }^{k}$ S. Shibata, ${ }^{e}$ J. Soni, ${ }^{d}$ K. Tanaka, ${ }^{f}$ F. Varsi ${ }^{d}$ and M. Zuberi ${ }^{a}$ [The GRAPES-3 Collaboration]

${ }^{a}$ Tata Institute of Fundamental Research, Homi Bhabha Road, Mumbai 400005, India

${ }^{b}$ Graduate School of Science, Osaka City University, Osaka 558-8585, Japan

${ }^{c}$ Aligarh Muslim University, Aligarh 202002, India

${ }^{d}$ Indian Institute of Technology Kanpur, Kanpur 208016, India

${ }^{e}$ College of Engineering, Chubu University, Kasugai, Aichi 487-8501, Japan

${ }^{f}$ Graduate School of Information Sciences, Hiroshima City University, Hiroshima 731-3194, Japan

${ }^{g}$ Institute for Space-Earth Environmental Research, Nagoya University, Nagoya 464-8601, Japan

${ }^{h}$ Institute for Cosmic Ray Research, Tokyo University, Kashiwa, Chiba 277-8582, Japan

${ }^{i}$ Utkal University, Bhubaneshwar 751004, India

${ }^{j}$ Indian Institute of Technology Jodhpur, Jodhpur 342037, India

${ }^{k}$ Indian Institute of Technology Indore, Indore 453552, India

${ }^{l}$ Dibrugarh University, Dibrugarh 786004, India

E-mail: 10medha.riya@gmail.com, pkm@tifr.res.in

The deflection of cosmic rays (CRs) in the interstellar magnetic field results in an almost isotropic flux as observed on Earth. However, an anisotropy has been observed at the level of $\sim 10^{-4}-10^{-3}$. The GRAPES-3 experiment located at Ooty, India consists of an array of 400 plastic scintillator detectors. It measures the particle densities and their relative arrival times in extensive air showers produced by the CRs. This information collected is then reconstructed to obtain the energy and direction of the primary CRs. The near-equatorial location of GRAPES-3 provides an opportunity to study this anisotropy in both hemispheres of the celestial sphere in the TeV-PeV energy range. However, detector and atmospheric effects that induce a few per cent change in the primary CR flux are challenges to be addressed. This work describes the use of the time scrambling method to address some these systematics and observe anisotropy.

$37^{\text {th }}$ International Cosmic Ray Conference (ICRC 2021)

July 12th-23rd, 2021

Online - Berlin, Germany

*Presenter 


\section{Introduction}

The origin, acceleration mechanism and propagation of cosmic rays (CRs) is a century-old mystery. CRs get deflected by the interstellar magnetic field while propagating to Earth. This results in an isotropic CR flux on Earth. However, some anisotropy is induced due to the diffusion of cosmic rays through random component of Galactic magnetic field or inhomogeneous distribution of sources [1, 2]. Several ground based experiments, such as, IceCube [3], HAWC [4], Tibet AS $\gamma$ [5], MILAGRO [6], have observed CR anisotropy of strength $\sim 10^{-4}-10^{-3}$ in the Northern and Southern skies in different energy ranges. Experiments, such as, IceCube have also observed the phase of anisotropy to flip at $100 \mathrm{TeV}$. GRAPES-3, being located close to the equator, has the advantage of studying anisotropy in the overlapping region of the two hemispheres. It can also provide good overlapping measurements in energy with other experiments as it covers a wide energy range from $10 \mathrm{TeV}-10 \mathrm{PeV}$. This work uses the time scrambling method [3, 7] to estimate the background and detect anisotropy.

\section{The GRAPES-3 experiment}

The GRAPES-3 (Gamma Ray Astronomy at PeV Energies Phase-3) experiment is located at Ooty $\left(11.4^{\circ} \mathrm{N}, 76.7^{\circ} \mathrm{E}, 2200 \mathrm{~m}\right.$ a.s.1.), India. The GRAPES-3 extensive air shower (EAS) array consists of 400 plastic scintillator detectors, each having an area of $1 \mathrm{~m}^{2}$ [8]. The scintillator array covers an area of $25000 \mathrm{~m}^{2}$ with the detectors arranged in hexagonal geometry, with an interdetector separation of $8 \mathrm{~m}$. Each of the scintillator detectors records the particle densities as well as the arrival times of particles in an air shower triggered within the GRAPES-3 array. The arrival times in each detector is measured precisely with a 32 channel high-performance time-to-digital converter (HPTDC) [9]. GRAPES-3 also has a $560 \mathrm{~m}^{2}$ tracking muon detector consisting of 3712 proportional counters (PRCs) [10]. GRAPES-3 has a nearly 100\% duty cycle and records around 3 million showers per day. A schematic of the GRAPES-3 array is shown in Figure 1.

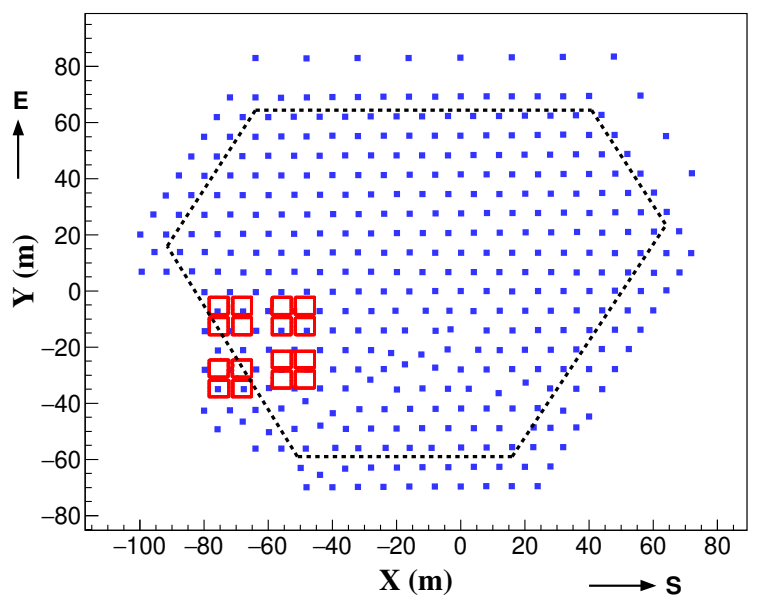

Figure 1: Schematic of GRAPES-3 air shower experiment 


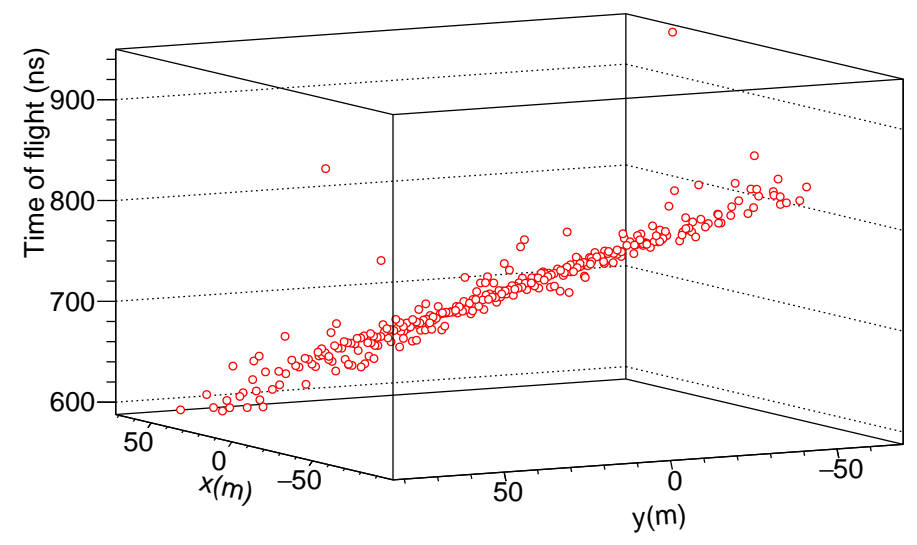

Figure 2: Arrival times of particles for a particular air shower

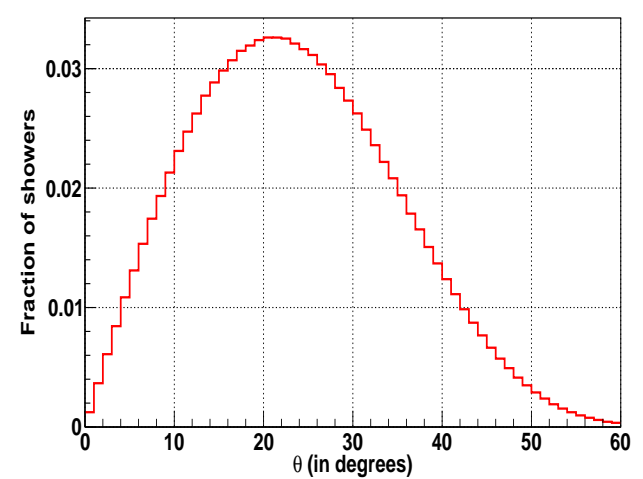

(a) Zenith distribution

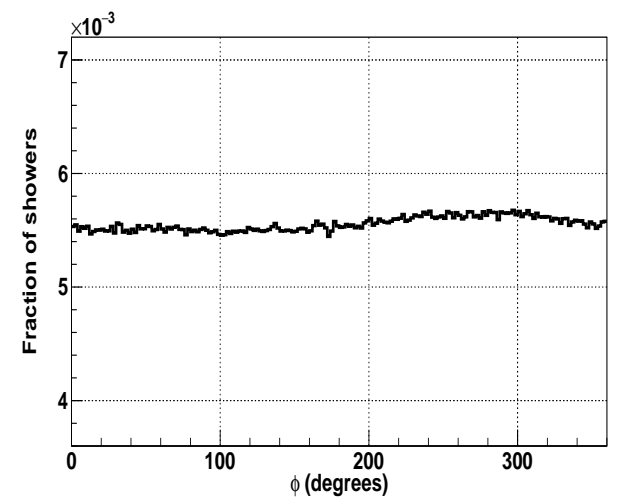

(b) Azimuth distribution

Figure 3: Distribution of local arrival directions

\section{Direction reconstruction}

The measured arrival times for a particular shower can be seen in Figure 2. A shower curvature correction is performed on the measured arrival times of the shower. A planar fit is performed on these corrected arrival times in order to obtain the direction of the shower. The shower curvature correction improves our angular resolution significantly from $1.3^{\circ}$ to $0.7^{\circ}$ at $>10 \mathrm{TeV}$ and from $0.5^{\circ}$ to $0.3^{\circ}$ at $>80 \mathrm{TeV}$ as described in [11]. The zenith $(\theta)$ and the azimuth $(\phi)$ distributions are shown in Figure 3.

\section{Analysis}

Three years of data starting from 1st January, 2014 upto 31st December, 2016 with a total of $3.4 \times 10^{9}$ events recorded in this period were used for this analysis. The following selection criteria were applied in order to select showers of interest for this analysis,

- $0^{\circ} \leq \theta \leq 60^{\circ}$ 


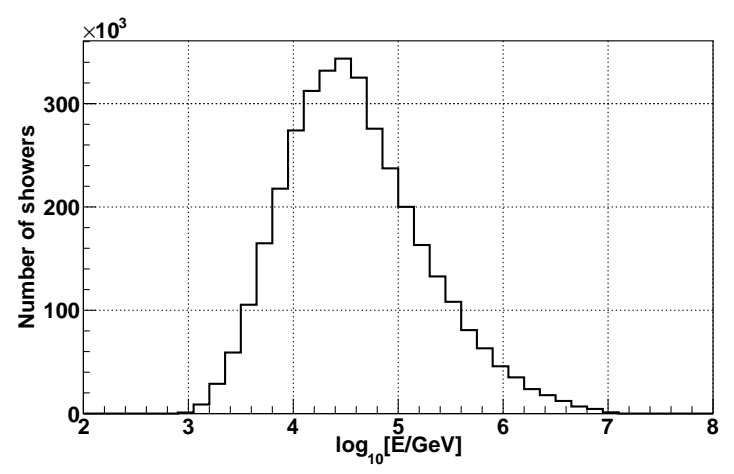

Figure 4: Energy distribution of selected events used for estimating median energy

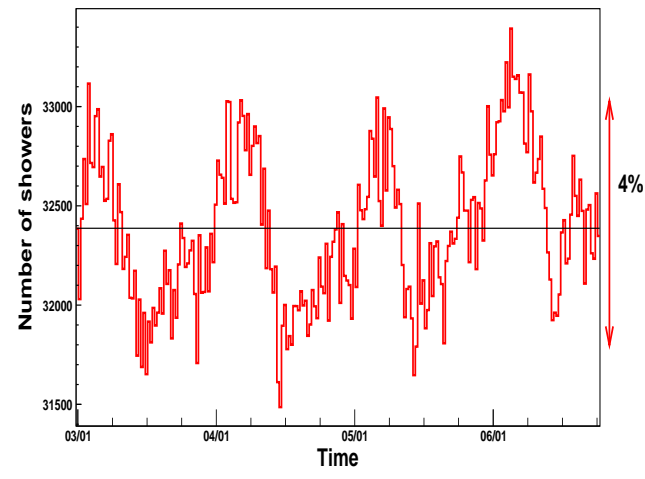

(a)

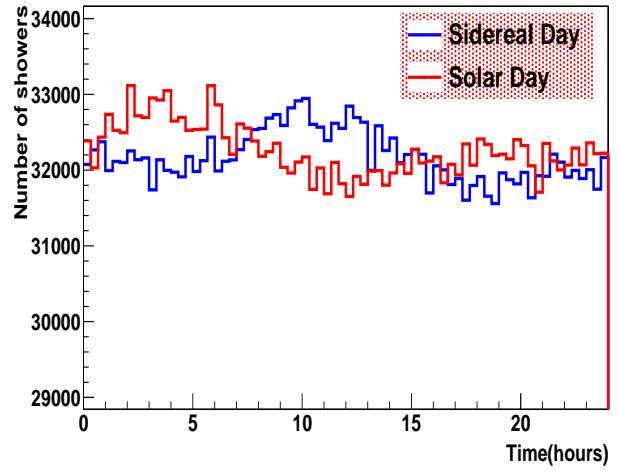

(b)

Figure 5: (a) Number of events collected in a 20 min time interval over a period of four days, (b) Variation in the number of events collected in 20 minute time interval for a solar and sidereal day

- $0^{\circ} \leq \phi \leq 360^{\circ}$

- Showers with proper angular reconstruction were used.

- More than 12 detectors should be triggered by the air shower.

After applying the above selection criteria, $2.49 \times 10^{9}$ events remain which were used for further analysis. The median energy for these remaining showers, estimated from simulation, is $28.18 \mathrm{TeV}$ (Figure 4). Atmospheric effects induce changes in the rate of events as shown in Figure 5 of around $2 \%$ from mean. There are also breaks in the data acquisition that lead to non-uniform exposure of the sky. The zenith and azimuth were converted to right ascension $(\alpha)$ and declination $(\delta)$ for all events satisfying the selection criteria, and a data map (Figure 6) was generated. To estimate the background, we use the time scrambling method described in [3, 7]. Each event is assigned a random event time which shifts its position in right ascension without changing the declination. This scrambles all astronomical anisotropic structures within the scrambling time window. For each recorded event, twenty fake events are generated and filled with proper weight in the reference map. Since the random times assigned to generate the fake events are selected from the recorded 


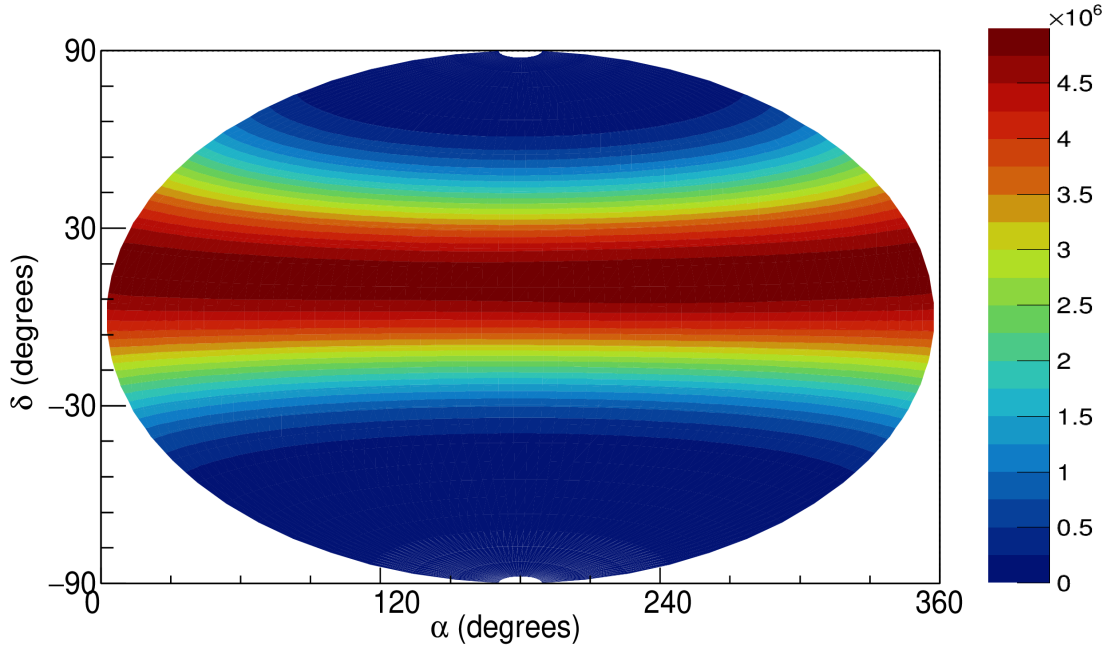

Figure 6: The data map: A wide declination range from $-40^{\circ}$ upto $60^{\circ}$ is covered, with the maximum number of events arriving from around $11^{\circ}$ declination

event time sample, breaks in the data acquisition are innately taken care of. The relative intensity between the data and scrambled maps gives us an estimate of the anisotropy. The anisotropy is calculated by,

$$
a=\frac{N_{i}-N_{r}}{N_{r}}
$$

where $N_{i}$ is the number of events in the $\mathrm{i}$-th pixel of the data map and $N_{r}$ is the number of events in the $\mathrm{i}^{\text {th }}$ pixel of the weighted scrambled map (Figure 7). The significance is calculated using the LiMa formula [12]. In this work, the scrambling time window was taken as $\Delta t=24 \mathrm{hrs}$ in solar time. The local arrival distributions and hence the acceptance of the detectors remain stable throughout a period of 24 hrs allowing us to search for large-scale structures on the Celestial sphere. The scrambling window of $\Delta t$ makes our search sensitive to a width of $15^{\circ} /$ hour $\times \Delta t$ in right ascension. Thus, a scrambling window of $24 \mathrm{hrs}$ makes our search sensitive to the largest existing anisotropic structures [3].

\section{Results and discussion}

Several small scale structures are observed which are consistent with the small-scale anisotropy observations from other experiments. The anisotropy map as shown in Figure 7 shows an excess around $45^{\circ}-80^{\circ}$ in right ascension and $-10^{\circ}$ to $25^{\circ}$ in declination with a strength of about $1.2 \times 10^{-3}$ and a significance of around $2.1 \sigma$ (Figure 8). This region is consistent with the region A structures observed by HAWC [13] and region 1 structures observed by ARGO-YBJ [14]. Another excess region is seen around $110^{\circ}-140^{\circ}$ in right ascension and $-30^{\circ}$ to $30^{\circ}$ in declination with a significance of about $1.2 \sigma$ which has some similarity with the region B structures seen by HAWC and region 2 seen by ARGO-YBJ. A faint deficit structure is also seen with a maximum strength of $6 \times 10^{-4}$ within $200^{\circ}-270^{\circ}$ in right ascension and $-15^{\circ}$ to $10^{\circ}$ in declination with a maximum significance of around $1.2 \sigma$. However, the large scale structures are not observed as they might be 


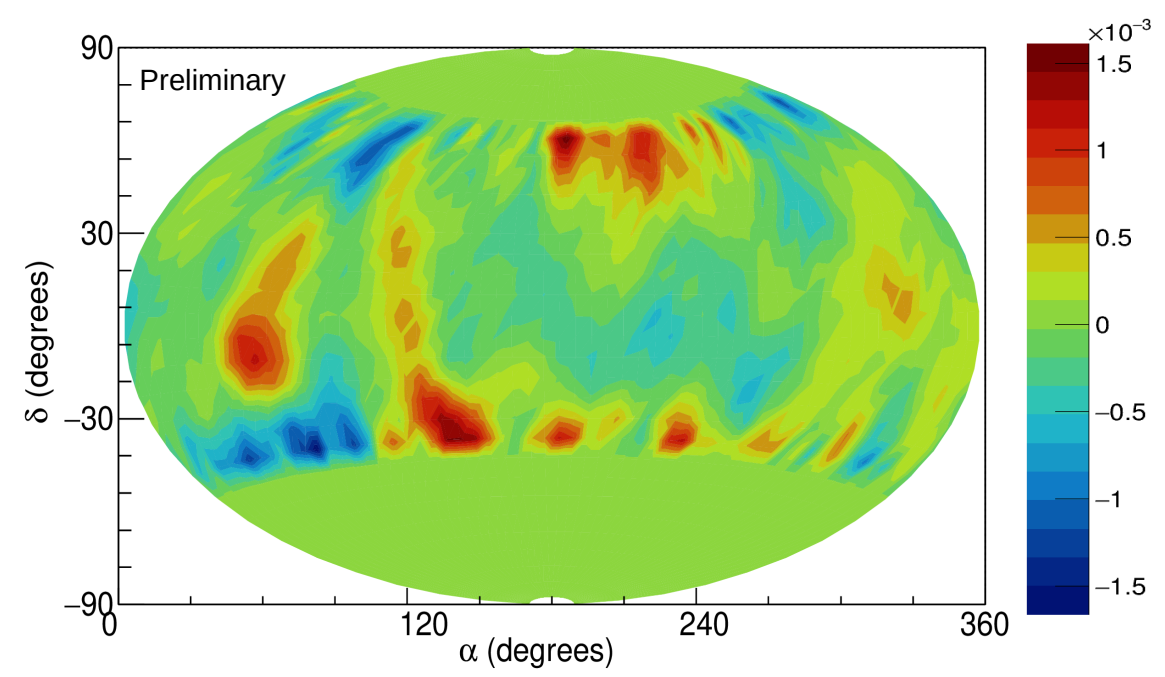

Figure 7: The relative intensity map : Several small scale anisotropic structures were observed

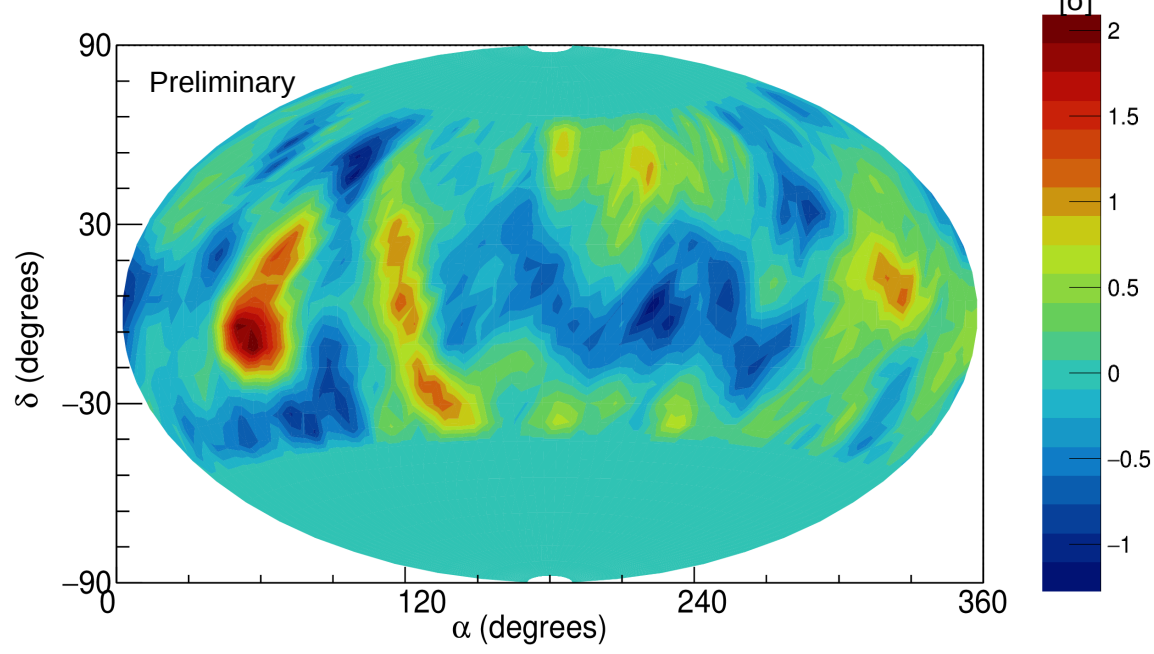

Figure 8: The significance of the observed anisotropy

getting suppressed due various systematic effects. Further analysis in progress, with higher statistics and a better understanding of various systematics, is expected to complement the observations from both the hemisphere experiments.

\subsection{Acknowledgements}

We are grateful to D.B. Arjunan, G.P. Francis, V. Jeyakumar, S. Kingston, K. Manjunath, S. Murugapandian, B. Rajesh, K. Ramadass, V. Santoshkumar, M.S. Shareef, C. Shobana, R. Sureshkumar for their role in the efficient running of the experiment. We would also like to thank Abhradeep Roy (TIFR) for his help with Celestial coordinate transformations. 


\section{References}

[1] Blasi, P. et. al., J. Cosmol. Astropart. Phys, 1201, 011

[2] Pohl, M. et. al., D. 2013, Astrophys. J., 766, 4

[3] M. G. Aartsen et al 2016 Astrophys. J. 826220

[4] A. U. Abeysekara et al., 2018 Astrophys. J. 86557

[5] Amenomori M et al. 2017 Astrophys. J. 836153

[6] A. A. Abdo et al 2009 Astrophys. J. 6982121

[7] Alexandreas et al., 1993, Nucl. Instr. Meth. Phys. Res. A, 328, 570

[8] S.K. Gupta et al., Nucl. Instr. Meth. A 540 (2005) 311-323

[9] S.K. Gupta et al., Exp Astron (2013) 35:507-526

[10] Y. Hayashi et al., Nucl. Instr. Meth. Phys. A 545 (2005) 643

[11] V.B. Jhansi et al., J. Cosmol. Astropart. Phys. 07 (2020) 024

[12] Li T \& Ma Y 1983 Astrophys. J. 272 317-324

[13] A. U. Abeysekara et al 2014 Astrophys. J. 796108

[14] B. Bartoli et al. , 2013 Phys. Rev. D 88, 082001 